\title{
Estimation of Physical Parameters of an Induction Motor Using an Indirect Method
}

\author{
Rui Esteves Araujo ${ }^{\text {, Member. IEEE, Américo Vicente Leite }}{ }^{2}$, and Diamantino Silva Freitas ${ }^{1}$ \\ 'Faculty of Engineering, Univ. of Porto, R. Dr. Robeno Frias.4200-465 Porto, Portugal, email : raraujo@fe.up.pt \\ ${ }^{2}$ Polytechnic Institute of Bragança., Quinta de Sta. Apolónia. Apartado 134, 5300 Bragança, Portugal
}

\begin{abstract}
In this paper a new method of estimation of the induction motor electric parameters is presented and discussed. The proposed method uses the indirect approach that consists in estimating the continuous-time parameters by first fitting a discrete-time model to the data and then converting this model to a continuous-time version. The main contribution is that by using a polynomial pre-fiter it is possible to compute the induction motor equivalent circuit parameters via an inverse transformation between the discrete-time and the continuoustime models. The procedure is potentially useful for the design of self-commissioning drives and may provide initial estimates to extended Kalman filtering type of procedures. The effectiveness of the proposed method is verified by simulated tests and results of the methad are discussed.
\end{abstract}

\section{INTRODUCTION}

Recent development in digital signal processing and power eiectronics makes it possibje to realise $\mathrm{AC}$ drives, which may cornpete with DC motors with respect to performance, cost and reliability. Therefore the technologies for digital signal processing control of induetion mators are developing rapidly and are the subject of research in universities as well as in industrial companies. Today's advanced control methods for inverter fed induction motor drives are based on the vector control approach [1]. Afthough the principles of the vector control method were already established in the early seventies by Blaschke [2], a real industriai application was only possible after the fast developments in power electronics and digital processors in the nineties. Vector control methods have been extensively researched in the past decades, but a few general problems still remain. According to a recent survey by Leonhard [3]. one of the more important aspects in research on induction motor drives should be a further improvement in the parameter estimation probiem of the induction motor. In this line of thinking. for example, in the vector control method of the induction motor, accurate knowiedge of the mignitude and position of the rotor flux phasor is necessary. In a standard induction motor no measure of the rotor current is available, what means that the rotor flux has to be estimated by an observer. which is based on the mathematical model of the induction machine. Additionally, the induction motor parameters vary with the operating conditions, the inductances tend to saturate at high flux levels and the resistances tend to increase as an effect of beating. In this way the problem of identification of the machine model is a precondition in order for these models to be used for reconstruction of machine quantities, such as flux or torque, from easily measurable quantities, such as terminal voltages and currents.

The conventional techniques for the estimation of the induction motor equivalent circuit parameters are based on the no-load and the blocked rotor tests. The main disadvantage is that the motor has to be locked mechanically and tests have to be carried out by skilled operators. Therefore, there is a need to identify the machine parameters automatically before the machine is started. In this paper, a new procedure is presented which can be used for automatic off-line identification of the electrical parameters from measurements of the stator voltages and currents. The experiments are performed when the motor is at standstill and the inverter of the drive is used to generate the signals required for the parameter estimation.

Several other methods of motor parameters estimation have been proposed in the literature $[4,5]$. in [4] the extended Kalman fither is formulated to allow the joint estimation of the state variables and motor parameters. In [5], the electrical parameters are estimated by using a linear regression model based on the continuous-time model of the induction moror. The same approach is used by Stephan and co-workers in [6], but with the model of the induction motor in the rotor coordinate frame.

Unlike these approaches, the present paper proposes a method that does not need handing of the time derivatives of the input signals. In order to preserve the model structure and the physical insight it is preferred to estimate the parameters directily in the continuous-ime mout. The different approaches to address this problem have been classified by Unbehauen and Rao [7] into two main classes:

- Indirect approach, where the continues-time model is transformed into a discrete-time mode), which is then submitted to identification. The desired parameters of the continuous-time model are then obtained by transferring the identified discrete-time model back into continuous-time.

- Direct approach, where the parameters of continuoustime models are determined by processing the samples of the input and outpin signals, without the need of identifying an internediate discrete-time model. The main idea of this approach is that parameters are obtained by processing the original differential equations often through the use of filters or others operators that are represented by a discrete-time approximation. The calculation of derivatives however can leact to serious problems if the signals are compted with 
noise or have steep waveforms. To avoid the calculation of derivatives, we will use an indirect method using a polynomial pre-fitter [8].

The contribution of this work is therefore the presentation of a new technique to estimate the induction motor parameters by a indirect method without need for differentiation. The method allows the estimation of the physical parameters of the induction motor fed by a voltage source inverter.

This paper is organized as follows. Section II discusses the structural identifiability of the standard induction motor model. Then, a equivalent model with only four unknown parameters is derived and discussed. Additionally, a formulation of the parameter estimation is given. The identification method is explained in section lll, Specifically. the polynomial pre-fitter is applied so that the estimation of continuous-time parameters can be determine by the inverse function. Results are given in section IV. Finally, in section V some conclusions are presented.

\section{ii. InDUCTION MACHNE MODEL AND Estimation} of Physical Parameters at STANDSTILI

It is crucial to start with a good and appropriate model of the induction motor when designing an algorithm for parameter estimation. Another important question in parameter estimation which should be considered is to know if is it possible to estimate all parameters in the model structure. This question concerns whether the model structure is identifiable or not, meaning whether the identification procedure will yield a unique value of the parameter or not.

\section{A. Structural Idenifiability of the inctuction Motor}

It is well know, see Ljung [9], that a model structure is not structurally identifiable if the model structute is overparameterized. Roughly speaking a necessary condition for a model to be uniquely identifiable is that there exists a unique parametrization, i.e. only one set of parameters can describe the same input-output behaviour of the model.

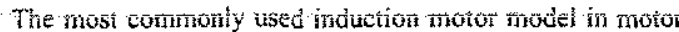
drives is the $T$ - form circuit shown in Fig. 1. For this model the flux-linkage of the stator windings $\left(\Phi_{\mathrm{s}}^{\mathrm{s}}\right)$ and the rotor windings $\left(\Phi_{\mathrm{s}}^{\mathrm{s}}\right)$ are, in case of a linear magnetic circuit, rejated to the winding currents $\left(i_{\mathrm{S}}^{\mathrm{s}}, i_{-\mathrm{r}}^{\mathrm{s}}\right)$ by the following equations:

$$
\begin{aligned}
& \Phi_{\mathrm{s}}^{\mathrm{s}}=\left(l_{\mathrm{s \sigma}}+l_{\mathrm{m}}\right) i_{\mathrm{s}}^{\mathrm{s}}+l_{\mathrm{m}} i_{\mathrm{r}}^{\mathrm{s}} \\
& \Phi_{\mathrm{r}}^{\mathrm{s}}=\left(l_{\mathrm{ro}}+l_{\mathrm{m}}\right) l_{\mathrm{r}}^{\mathrm{s}}+l_{\mathrm{m}} i_{\mathrm{s}}^{\mathrm{s}}
\end{aligned}
$$

where subscripts $s$ and $\mathrm{r}$, stand for stator, and rotor values, $l_{\mathrm{m}}$ magnetizing inductance, $l_{s e}$ and $l_{r \sigma}$ are leakage inductance for stator and rotor, respectively.

The induction motor described with 3 inductances can be modelled by an infinite number of different parameters

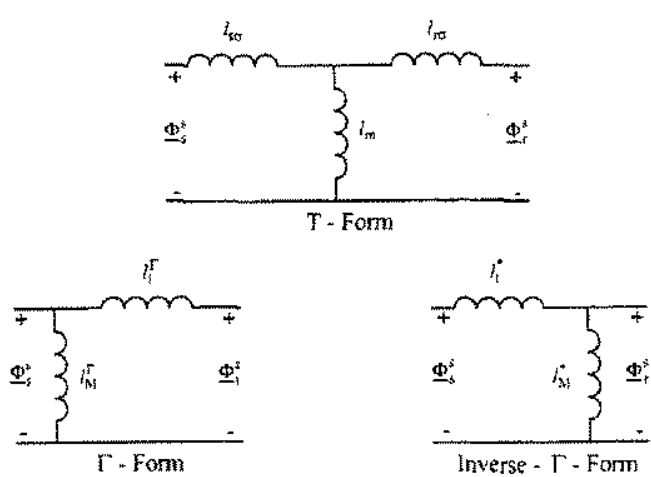

Fig. I Equivalent flux linkage models with identical input impedance.

values. There is one degree of freedom in the model definition because the three depend inductances can be described by two independent inductances. This means that the flux linkages with three inductances are not uniquely parametrized and hence not uniquely identifiable. Therefore the $T$ - form model is not structurally identifiable.

This difficulty is solved by the use of a simple change of variables that permits to obtain two mathematically equivalent models with two inductances only. In [10] the $T$. form circuit is transformed into $\Gamma$ - form and inverse $\Gamma$ circuits without loss of parameters information and accuracy (see Fig. 1). A systematic analysis of all possible structures is also presented by Yamamura [11].

\section{B. The Choice of Model}

The standard set of equations (1) relating statot and rotor currents and flux linkages have one redundant parameter and can be reartanged into a set of equations with only one leakage inductance. $l_{1}^{*}$ (in this case we have $l_{\text {ro }}=0$ ). The corresponding equations for this inverse $\Gamma$ model with only one leakage inductance are:

$$
\begin{aligned}
& \Phi_{\mathrm{s}}^{\mathrm{s}}=l_{l_{\mathrm{s}}}^{*}+i_{\mathrm{M}}^{*}\left(i_{\mathrm{s}}^{\mathrm{s}}+i_{-\mathrm{r}}^{\mathrm{s}}\right) \\
& \Phi_{\mathrm{r}}^{\mathrm{s}}=l_{\mathrm{M}}^{*}\left(i_{\mathrm{s}}^{\mathrm{s}}+i_{\mathrm{r}}^{\mathrm{s}}\right)
\end{aligned}
$$

Thus, with the previous assumptions, and the hypothesis that the stator and rotor windings are electrically and magnetically symmetrical, the air-gap is uniform. the field distribution is sinusoidal, and the effects of magnetic saturation, eddy-currents and hysteresis are neglected. the induction motor is represented by its state-space model in a fixed reference frame, which is also known as the inverse $r$ :

$$
\left\{\begin{array}{l}
\dot{\dot{x}}=\underline{A}\left(\omega_{\mathrm{m}}\right) \underline{x}+\underline{B} \underline{u}_{\mathrm{s}}^{\mathrm{s}} \\
\dot{v}_{\mathrm{s}}^{\mathrm{s}}=\underline{C} \underline{x}
\end{array}\right.
$$

where the state variables are the stator current space vector and rotor flux space vector, $x=\left[\begin{array}{ll}i_{-s}^{s} & \Phi_{r}^{s}\end{array}\right]^{T}$. 
At this point it is quite interesting to interpret the discretization process as a mapping or a transformation, i.e. the discretization may be viewed as a mapping from the $\mathcal{D}_{c}$ domain to the $\mathcal{D}_{d}$ domain. Suppose we have two sets $\mathcal{K}_{c}$ and $\mathscr{M}_{d}$. Suppose additionally that we can define a function $F$ which assigns, to each element in the set $\mathcal{M}_{c}$ one element of the set $M_{d}$. In other words, $F$ is a function which transfoms, or maps, $\mathcal{D}_{c}$ into $\mathcal{D}_{d}$ and we will denote this by $F: M_{c} \subset \mathfrak{R}^{h} \rightarrow \mathscr{M}_{d} \subset \Re^{\prime}$ such that

$$
\theta_{d}=F\left(\theta_{c}\right)
$$

which can be determined from mathematical modelling, for example with Maple.

After establishing the relation between the continuous-time and discrete-time models, the discrete parameters $\theta_{t}$ will be estimated by a standard identification algorithm $A$ and a set $Z$, i.e. $\hat{\theta}_{d}=\mathscr{A}(Z)$. For example, an identification algorithm with an output-error method can be used. The orders of the polynomials of the output-error model are defined by the structure of $G_{\alpha}$, which may be known in advance based on the continusous-time to discrete-time transformation

We have prepared in this subusection the basis for the crucial step of conversion of parameters from the discretetime domain to the continuous domain. We consider that, given a continuous-time model for estimation of its parameters, under some reasonable assumptions, it is possible to reformulate the problem in a way that permits the straight forward application of discrete-time black-box identification using, for example, the routines available in the System Identification Toolbox of Matlab [12]. It is quite obvious that, if the discretentime model is not accurate, the equivalen continuous-time model will also be inaccurate. so, a number of precautions must be considered in this step.

We now turn our attention to study the possibility of conversion, that is, the calculation of the coefficients of the differential equation model according to the function to be found that performs the mapping between the continuous-time domain and the discreteme domain This is the subiect of the next sub-section.

\section{B. The Basic Difficulty}

We now come to the topic of inversion of function $F$ (see (10)). It is a small but extremely important step in our problem. Roughly speaking, a function $F: A \rightarrow B$ is invertible if there exists a function $G: B \rightarrow A$ such that $F G$ and $G F$ are the identity mappings on the sets $A$ and $B$, respectively and we will denote it by $F^{-1}$. Let us make this idea more precise in our case. Based on the estimated discrete-time parameters $\hat{\theta}_{d}$, the continuous-time parameters $\hat{\theta}_{c}$ have to be determine by the inverse function given by

$$
\hat{\theta}_{c}=F^{-1}\left(\hat{\Theta}_{d}\right)
$$

when the inverse exists, so giving an unique solution.
The main difficulty with finding this inverse function is associated with the mapping of the zeros of the discrete-time model transfer function to the continuous-time model. It is well known that with a zero-order hold approach for signal handling. the mapping between the discrete-time poles and their continuous-time counterparts is reasonably simple, because these are mapped by means of complex exponentials. However, this is not the general case for the zeros, for which no general closed form equations exist, only approximate expressions; see for instance [13], [14] and [15]. Consequently, a poor parameter mapping will usually occur affecting the accuracy of the final continuous-time parameter estimation.

It is of fundamental importance to keep in mind that the poles of a system describe its natural response when the input signal is zero (zero-state response). Obviously. then, the intersample behaviour cannot affect the natural response of the system and therefore it has no effect on the poles of the system. Such a simple and elegant situation does not hold for the zeros of a system which contribute 10 describe its behavior when a non zero imput signal is present. The zeros will be intimately related to the intersample behavior used for the discrete-time model, and there will be no simple mapping from continuous-time to discrete-time zeros. In the present case the use of the bilinear transfomation introduces an integration type of intersample behaviour.

\section{Computing the Parameter Vector $\hat{\theta}_{c}$}

The main problem to resolve appears with the transfer function zeros. The solution described in [8] uses a polynomial pre-filter applied to the input signal that guarantees that the continuous-time parameters could be uniquely determined form the discrete-time parameters. The rationale of this solution to the problem of obtaining unique continuous-time from the discrete-time parameters is based on the fact that the poles and finite zeros map to the $z$ plane according to

$$
z=\frac{1+s \frac{T}{2}}{1-s \frac{T}{2}} .
$$

Furthemore, the zeros at infinity in the $s$-plane map into zeros at $z=-1$. In this line of thought the measured input is filtered through the polynomial filter for input data, given as follows:

$$
u^{*}(k)=\left(1+q^{-1}\right)^{r} u(k)
$$

where $q^{-l}$ is the backward shift operator, $r$ is the relative degree of the model rational function and $u(k)$ is the input data sample at instant $k$.

At this stage, we assumed to have the discrete transfer function computed according to (9) and a set of input-output data, $z$. The expression (13) implies that each input-output measurement defines a new set denoted by $z^{*}$ containing $i^{\circ}$ : 
The input and output variables are, respectively, the stator voltage space vector $\left(\underline{u}_{5}^{s}\right)$ and current space vector with

$$
\begin{aligned}
& A\left(\omega_{\mathrm{m}}\right)=\left[\begin{array}{cc}
\frac{r_{\mathrm{s}}+r_{i}}{l_{l}^{*}} & \frac{\mathrm{I}}{i_{j}^{*}}\left(\frac{r_{\mathrm{I}}}{l_{\mathrm{M}}^{*}}-\mathrm{j} \omega_{\mathrm{m}}\right) \\
r_{\mathrm{r}} & \left(-\frac{r_{\mathrm{r}}}{l_{\mathrm{M}}^{*}}+\mathrm{j} \omega_{\mathrm{m}}\right)
\end{array}\right] \\
& B=\left[\begin{array}{c}
1 \\
f_{1}^{*} \\
0
\end{array}\right] \\
& \underline{C}=\left[\begin{array}{ll}
1 & 0
\end{array}\right]
\end{aligned}
$$

where $a_{3,}$ is the mechanical angular velocity of the rotor. Note that all variables are handled in the stator coordinate system $(\alpha \beta \beta)$.

\section{Parameter Estimation Problem}

From the set of first-order non linear differential equations of (3). when $\omega_{3 r}$ is constant the coefficients of $\left.A(\omega)_{m}\right)$ are constant. Therefore (3) can be written as a set of linear differential equations. In this condition it is possible to find the transfer function of the induction motor through the following equation:

$$
Y_{m}\left(s, \theta_{m f}\right)=\frac{i_{s}^{\mathrm{s}}}{\underline{u}_{s}^{s}}=\underline{C}(s I-\underline{A})^{-1} \underline{B} .
$$

Now, the model of an induction motor at standstill is described by the following transfer function:

$$
\begin{aligned}
Y_{m}^{r}\left(s, \theta_{m}\right) & =\frac{i_{s}^{5}}{\underline{u}_{s}^{s}} \\
& =\frac{l_{\mathrm{M}}^{*} s+r_{t}^{*}}{l_{\mathrm{M}}^{*} l_{i}^{*} s^{2}+\left(r_{s} l_{\mathrm{M}}^{*}+l_{l}^{*} r_{r}^{*}+l_{\mathrm{M}}^{*} r_{r}^{*}\right) s+r_{s} r_{Y}^{*}}
\end{aligned}
$$

where $\underline{H}_{\mathrm{s}}^{\mathrm{s}}$ is the stator terminal voltage as input signal and stator terminal current $i_{-s}^{\text {s }}$ as output signal. The transfer function representes the machine admitlance and is parametrized by physical parameters. denoted by the parameter vector $\theta_{m}=\left[r_{s}, l_{i}^{*}, l_{\mathrm{M}}^{*}, r_{\mathrm{r}}^{*}\right]^{r} \in \Re^{4}$. The circuit representation of this model is given in Fig. 2.

The relation between the parameters of the equivalent circuit in $T$ - form shown in Fig. 1 and the parameters of circuit ita Fig. 2 are as follows:

$$
\begin{aligned}
& l_{1}^{*}=0 l_{\mathrm{s}} \\
& l_{\mathrm{M}}^{*}=\frac{l_{\mathrm{m}}^{2}}{l_{\mathrm{r}}} \\
& r_{\mathrm{r}}^{*}=\left(\frac{l_{\mathrm{m}}}{l_{r}}\right)^{2} r_{\mathrm{r}}
\end{aligned}
$$

In the next step we can define the following parameter estimation problem: Consider an induetion motor at standstil described by its transfer function (6) with unknown parameters $r_{s}, l_{1}^{*}, l_{M}^{*}, r_{f}^{*}$. Available are the sampled data sequences of the stator voltages and stator currents. The probjem is to determine the motor parameters $r_{\xi}, i_{i}^{*}, t_{\mathrm{M}}^{*}, r_{\mathrm{r}}^{*}$. To avoid the calculation of derivatives, we will use an indirect method using the bilinear transformation. A summary of this approach is outlined next.

\section{INDIRECT METHOD TO PARAMETER ESTIMATION}

In this section, we present a solution based on an indirect approach to the above stated parameter estimation problem. This consists of two steps. In the first step a discrete-time model is estimated from the measured input and output signals. Once these parameters are determined, the coefficients of the differential equation can be computed via a mapping function between the discrete-time model and the continuous-time model.

\section{A. From Conthwous to Discrete Tine}

The first step consists in establishing the relation between the continuous-time model and an equivalent discrete-time model. It is assumed that the model, and the measurement data obtained from this system, allow a description [9] like the following:

$$
y_{m}(t)=G_{c}\left(p, \theta_{c}\right) z(t)+e(t)
$$

where $p$ is the differentiation operator, $G_{r}$ is the system, $y_{m}(t)$ is the measured output signal, $z(t)$ is the input signal and $e(t)$ is aun audutive stationaly winte notse.

In order to establish the model structures for the systen, we need to transform the transfer function $G_{c}$ into a discrete-time model by means of the bilinear transformation:

$$
T: G_{d}\left(\Sigma, \theta_{d}\right)=\left.G_{c}\left(s, \theta_{c}\right)\right|_{s=\frac{2}{T} \frac{z-1}{\Sigma+1}} .
$$

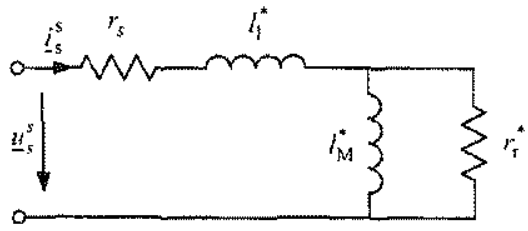

Fig. 2 Equivalent circuit in Inverse $\mathrm{T}$ form. 


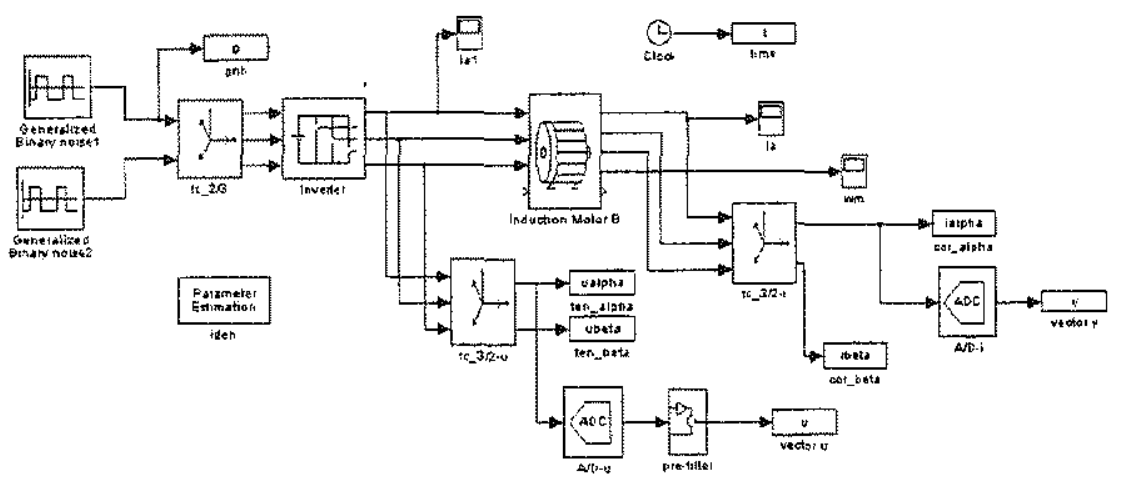

Figute 3 - Simulation diagram for the identification procedure.

\section{B. Numerical Hllustration}

The performance of the identification method illustrated in figure 3 was investigated by means of simulations done using the Vector Control Signal Processing Blockset for Simulink environment [19].

in order to evaluate the performance of this methodology, the following measure of estimation error was used:

$$
\varepsilon=\frac{\left|\theta-\theta_{0}\right|}{\mid \theta_{0} \|}
$$

where $\theta_{0}$ is the true parameter vector and $\theta$ is the estimated parameter vector

Simulations were performed with the objective of evaluating the proposed method. We considered two simulations, which differ only in the use of two different motors ( $A$ and $B$ ). The respective physical parameters established in accordance with the physical model represented in the equivalent circuit of Fig. 2 are listed in table !.

The results collected in table 11 summarize the parameter estimations obtain with simulations. The parameter error nom in indicative of the accuracy of estimation, in generol the results show that in all cases it is possible to obtain quite good estimates of the parameters. However. these results are presented without noise, in the future results will be presented with noise added to the output signal.

\section{CONCLUSHONS}

We have studied the problem of parameter estimation of an induction motor based on an inditect approach. This methodology involved a first phase identification of the parameters of a discrete-time model and secondly a transformation of the corresponding parameters to the physical parameters. Numerical test of the identification algorithm exhibits a good accuracy. As this research is in a preliminary phase, further work is needed. Future works will consist in expanding the simulation studies with noise added to signals and a practical implementation.

\section{REFERENCES}

(11) Vas, P.. Electrical Machines and Drives. Oxford Science Publications. New: York, 1992

[2] Blaschike, F." "The Principle of Fieid Orientation as Applied to the New Transvektor Closed - Lopp Control System for Rotasing Field Machines". Siemens Rev, wol. 34, pp. 217-220, May, 1972

(3) Leonhard, $W$. "Controlled $A C$ drives, a successtul transition fron iceas to industrial practice". Control Enyineerimg Practice, vol, 4. no7. pp. $897.908 .1990^{\circ}$

[4] Loron, L.. Laliberté.G., "Applicarion of the extended kalman filter to parameters estimation of induction morors , in Proceedings of the the Earopean Poster Electronics Assuciation. pp. 85-90. 1993.

55] Moons, $C$. Moor, B.. "Parameter identification of induction motor drives". Aztonatica. vol. $31 . n^{\circ} 8$. pp. 1137-1147, 1995.

(6) Stephan. J.. Bodson. M., Chiasson. J., "Reat-fime estimation of the paranteters and fluxes of induction molors". IEEE Tranisactions on industry applications, vol. 30. $n$ 3. May/june. pp. 746-759, 1994.

[7] $\mathrm{H}$. Unbehauen and G. P. Rao, "A review of idemtification in continwous-time systems," Ammal Reviests in Conmol, vol. 22, pp. $145 \div 171.1998$

[8] Ruj Esteves Araujio. Modelacào, destifficaçào e comirolo do motor de indiłçáo rrifásico. PhD Thesis (in Ponuguese). FEUP. 200).

[9] L. Ljang. Sistem Identification : Theon for the User. 2nd edilon. Prentice Hall, New Jersey, 1999.

[10] Siemon. G.R.. "Circuit models for polyphase induction machines". Electic Mochines and Power Sistems. vol. 8. pp.369-379. 1983.

[11] S. Yamamura. AC Motors for High Performance Applications. Marcel Dekker. 1986.

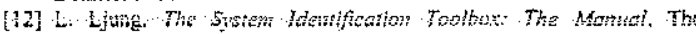
Math Works Inc., 4th edition. Natick. MA. 1995.

[13] K. J. Áström. P. Hagander and J. Sternby." Zeros of sampled systerns". Atfomatica. vol. 20. nô. 1, pp. $31 \cdot 38.1984$

114] M. 3. Blachuta, "On zeros of transfer functions," IEEE Trans. Anomatic Control. vol. 44. no. 6. pp. \{229.1234. 1999.

[15] B. M. Chen and S. R. Weller. "Mappings of the finite and infinite zero structures and inveribility structures of general linear mutivariable systems under the bilinear transformation", Automatica. vol. 34, no. 1 . pp. 111-124. 1998

[16] S.C.L jayme, "Identification des Paramètres des Machines Electriques à Courant Alternatif". Thèse de Docteur de L'INP de Toulouse, 1995.

[17] A, V. Leite, Aplicaçâo de técnicos de modelitaç̧ầo paramétrico identificacáo ao motor de induçào rrifósice. Mastet Thesis (in Portaguese KEUP. 1996.

[18] H.J.A.F. Tulleken, "Generalized binary noise test signal concept for improved idemification experiment design", Auromatica, vol. 26 , $\mathrm{nt}^{\circ} \mathrm{i}$, pp. $3-49,1990$

[19] R. E. Araújo and D. S. Freitas, "The development of vector control signal processing blocksei for simulink: philosophy and implementation." Proceedings of the $24 \mathrm{th}$ Ammial Conf. of the IEEE industrial Electronics Socien. Aachen. Gemany, 1998 


$$
z^{*}=\left\{u^{*}(k), y_{m}(k) ; k=0, \ldots, N-1\right\} .
$$

So, assuming that the conditions established by the inverse function theorem are all fulfilled, the parameter vector $\theta_{c}$ is calculated by

$$
\overbrace{\hat{\theta}_{c^{\prime}}=F^{-1}\left(\hat{\theta}_{d}\right)=}^{\text {inversion }}=\overbrace{\lim _{r \rightarrow 0} \lim _{\hat{r} \rightarrow \infty} \hat{\theta}_{d}=\theta_{d}}^{\text {estimation }}
$$

where $\theta_{d}$ is estimated by the identification algorithm $\mathcal{A}$ using input-output data $Z^{*}$, assuming that the estimation is close to the true value of the parameter vector for large data sets and small sampling period.

\section{Simulation Stumy}

\section{A. Jdentification Procedure}

One of the difficulties found in the literature [16] and corrobotated in the work of Vicente Leite [17] comes from the loss of persistence of the rypical signals fed to the induction motor in normal running. With effect, the rypical signals are rich in hamonics, its harmonics content are in a zone of high frequencies (the order of some units or few tens of $\mathrm{kHz}$ ), without practical interest for the identification of the physical parameters of the moror.

The quality of the identification depends, to a greal extent. on the exciting input signal form, frequency and observed time). In fact. the performance depends on the richness of the excitation signal, that unfortunately cannot aiways be guaranteed under normal conditions of operation. We have introduced in this work a Generalized binary noise moposed by Tulleken [18] as input signal during the estimation experiments, that leads up to an optimal trajectory for identification.
The problem that has been enunciated in section 2 will be resolved through the methodology proposed in section 3 . So, when the transfer funcrion (6) is discretized by the bilinear transformation and taking in account that relative degree is 1 , the filtering will be:

$$
\underline{u}_{s}^{*}(k)=\left(1+q^{-1}\right) \underline{u}_{s}^{s}
$$

that results in:

$$
G_{d}^{*}\left(z, \theta_{d}\right)=\frac{\underline{I}_{s}^{s}(z)}{\underline{U}_{s}^{\mathrm{s}}(z)}=\frac{b_{1}^{d}+b_{0}^{d} z^{-1}}{1+a_{1}^{d} z^{-1}+a_{0}^{d} z^{-2}}
$$

in which the vector of the parameters of the discrete domain is:

$$
\theta_{d}=\left[b_{1}^{d}, b_{0}^{d}, a_{1}^{d}, a_{0}^{d}\right]^{T} \in \mathfrak{R}^{4}
$$

The estimates of the physical parameters are then calculated from the vector of parameters of the discrete-time domain on the basis of the following expressions:

$$
\hat{\theta}_{m}=\left[\begin{array}{c}
r_{s} \\
r_{1}^{*} \\
i_{\mathrm{M}}^{*} \\
r_{\mathrm{T}}^{*}
\end{array}\right]
$$

$$
=F^{-1}\left(\theta_{d}\right)=\left[\begin{array}{c}
\frac{a_{0}^{d}+a_{1}^{d}+1}{2\left(b_{1}^{d}+b_{0}^{d}\right)} \\
\frac{\left(1-a_{1}^{d}+a_{0}^{d}\right) T}{4\left(b_{1}^{d}-b_{0}^{d}\right)} \\
-\frac{\left(a_{0}^{d} b_{1}^{d^{2}}+b_{0}^{d^{2}}-a_{1}^{d} b_{1}^{d} b_{0}^{d}\right) T}{b_{1}^{d}-b_{0}^{d^{2}} b_{1}^{d}+b_{0}^{d} b_{1}^{d^{2}}-b_{0}^{d^{3}}} \\
-2 \frac{a_{0}^{d} b_{1}^{d^{2}}+b_{0}^{d^{2}}-a_{1}^{d} b_{1}^{d} b_{0}^{d}}{\left(b_{1}^{d}-b_{0}^{d}\right)\left(b_{1}^{d^{2}}-b_{0}^{d^{2}}\right)}
\end{array}\right]
$$

TABLE 1

MACHINE PARAMETERS

\begin{tabular}{ccccc}
\hline Notor & $R_{\mathrm{s}}(\Omega)$ & $L_{\mathrm{H}}^{*}(\mathrm{H})$ & $L_{\mathrm{M}}^{*}(\mathrm{H})$ & $R_{\mathrm{f}}^{*}(\Omega)$ \\
\hline $\mathrm{A}$ & 0.8 & 0.0113 & 0.0947 & 0.5497 \\
$\mathrm{~B}$ & 5.5 & 0.0446 & 0.3414 & 3.025 \\
\hline
\end{tabular}

TABLE II

ESTIMATED MACHINE PARAMETERS

\begin{tabular}{ccccccc}
\hline Motor & axis & $R_{s}(\Omega)$ & $L_{i}^{*}(\mathrm{H})$ & $L_{\mathrm{M}}^{*}(\mathrm{H})$ & $R_{\mathrm{Y}}^{*}(\Omega)$ & $\begin{array}{l}\text { Error } \\
\text { Norm }\end{array}$ \\
\hline $\mathrm{A}$ & $\alpha$ & 0.802 & 0.012 & 0.095 & 0.5443 & 0.0056 \\
$\mathrm{~A}$ & $\beta$ & 0.7989 & 0.012 & 0.0947 & 0.5428 & 0.0073 \\
$\mathrm{~B}$ & $\alpha$ & 5.5 & 0.049 & 0.3386 & 3.009 & 0.0043 \\
$\mathrm{~B}$ & $\beta$ & 5.502 & 0.049 & 0.3403 & 2.982 & 0.0068 \\
\hline
\end{tabular}

